\title{
La FMH sur le banc des accusés
}

Nous publions dans ce numéro la plainte contre l'introduction du TARMED déposée par la fédération des médecins prestataires de gestes techniques, chirurgicaux, interventionnels et radiologiques (FMS), de même que la liste des plaignants. Cette action en justice appelle les constatations suivantes:

1. Le Comité central de la FMH (CC) s'en tient à l'introduction, au $1^{\text {er }}$ janvier 2004, des tarifs TARMED dans le domaine de la LAMal. En outre, il ne voit pas de raison, à l'heure actuelle, de dénoncer la convention applicable au domaine AA/AM/AI.

2. Le CC continuera à poursuivre son objectif d'améliorer constamment la structure tarifaire TARMED, même si la deuxième phase de remaniement (RE II) n'a pas conduit aux résultats escomptés.

3. Le CC continue en particulier à prôner la création d'un tarif pour les médecins agréés fondé sur le TARMED. Cette option est la seule qui permettra de résoudre les problèmes des médecins agréés: une position défendue par le CC et par la FMH depuis 1993!

4. Les plaignants et leur entourage en appellent à la protection des minorités, un idéal profondément ancré dans les valeurs démocratiques de notre pays. La vérité est que pendant toutes ces années de négociations, les intérêts des minorités, aussi infimes soient- elles, ont été analysés et pris en compte dans la structure tarifaire TARMED. En revanche, il n'est pas acceptable qu'une minorité veuille maintenant l'emporter sur la majorité clairement exprimée lors de la votation générale de 2002. Quiconque agit ainsi s'expose à subir le reproche d'abus des institutions démocratiques.

5. Il n'y a pas lieu de s'étendre ici sur la teneur et la légitimité de la plainte de la FMS. Ne fûtce que pour des raisons de procédure, le CC a pour principe de ne jamais s'exprimer au sujet d'actions judiciaires en cours. Cependant, il est d'ores et déjà possible d'estimer précisément les dommages collatéraux que nous vaudra cette plainte: outre les frais de procès qui se monteront à des dizaines de milliers de francs prélevés sur les cotisations de membres, force est de constater qu'une fois de plus, le corps médical se retrouve l'otage des médias suite aux agissements d'une petite minorité obéissant à des motifs en vérité très clairs. Une situation devenue absolument intolérable.

Quoi qu'il arrive, ceci nous vaudra l'honneur peu commun de nous retrouver sur le même banc des accusés que ... la Confédération suisse ellemême.

Dr H. H. Brunner, président de la FMH 